\title{
LAS IDEAS DE LOS ALUMNOS Y ALUMNAS ACERCA DE LA ESTRUCTURA CELULAR DE LOS SERES VIVOS
}

\author{
CABALLER, M. J. y GIMÉNEZ, I. \\ Centros del Profesorado de Godella y Valencia.
}

\section{SUMMARY}

In this article we analyse the spontaneous ideas of students of different levels (EGB, BUP, Teacher Training), in relation to the concept of pluriceilularity. The results obtained show the low level of input of academic intruction in the teaching of the cellular structure of living creatures, despite the fact that this subject is treated repeatediy in primary, secondary and teacher training school.

\section{INTRODUCCIÓN}

En los últimos años se ha prestado considerable atención al estudio de las ideas que, sobre los conceptos científicos, poseen los y las estudiantes.

Se dispone ya de muchos resultados tanto en el campo de la Física y Química (Carrascosa 1985) como últimamente en el de Biología (Jiménez Aleixandre 1987) (Giordan, Martinand 1988), que muestran cómo las personas tienen arraigadas ideas acerca de los fenómenos naturales, bastante distintas de las explicaciones que se les da en la escuela al respecto.

Estas ideas o «concepciones espontáneas» pueden ser muy resistentes al cambio; por ello, una de las líneas más importantes en las investigaciones sobre didáctica de las ciencias ha sido, precisamente, el estudio de las causas de las ideas previas y su persistencia.

En el transcurso de su ejercicio docente muchos profesores y profesoras han detectado numerosas dificultades en el aprendizaje que ahora podríamos atribuir a la existencia, en la población escolar, de esquemas conceptuales que se comportan como obstáculos a la hora de aprender. Conocer las características de estos obstáculos se convierte en algo muy importante porque puede permitir la acción đocente y por tanto la calidad del aprendizaje.

En Biología se han realizado hasta el momento estudios de los esquemas conceptuales del alumnado en algunos temas, como por ejemplo, el concepto de generación espontánea (Giordan 1978), la constitución de los seres vivos (Brumby 1979), la estructura del aparato digestivo (Giordan 1982), fotosíntesis (Gené 1984, Cañal 1986), etc.

Nuestro trabajo se enmarca en la línea de estos estudios, y se dirige a la determinación de las ideas del alumnado sobre el concepto de pluricelularidad.

\section{PLANTEAMIENTO DEL PROBLEMA}

Uno de los conceptos básicos en Biología es la estructura celular de los seres vivos. Este es un concepto difícil de entender, puesto que no se puede derivar de la evidencia o de la observación cotidiana.

Podríamos plantearnos numerosas cuestiones en torno al aprendizaje de estos conceptos:

¿Se produce verdaderamente el aprendizaje significativo del concepto de célula como unidad de vida a to largo de la escolaridad obligatoria?

¿Comprenden ios alumnos y las aiumnas, de Ios diferentes niveles obligatorios, la complejidad de una estructura pluricecular de los seres vivos? 
¿Qué características tienen las ideas previas de los y las alumnas respecto de este tema?

¿Dónde puede residir el obstáculo epistemológico fundamental que tendremos que abordar (en el diseño deI plan de trabajo como docentes) para facilitar el aprendizaje de estos conceptos?

Una respuesta a estos problemas requiere un estudio complejo sobre diferentes aspectos parciales y concretos. Para poder realizarlo es necesario acotar el campo de investigación.

Así hemos escogido sólo un aspecto, que desđe nuestro punto de vista consideramos previo a cualquier otro estudio sobre el tema:

¿Cuáles son las características generales de las ideas de los y las alumnas sobre la constitución celular de los seres vivos?

\section{HIPÓTESIS DE TRABAJO}

Ciñéndonos al tema que hemos elegido (derivado del planteamiento inicial del problema), sabemos que existe entre los docentes-como consecuencia de su experiertcia en el aula-la idea de que el alumnado conoce y comprende que los seres vivos están formados por céluias.

Por otra parte, también son muy conocidas por el profesorado las dificultades que se plantean en el aula a la hora de comprender la fisiología de los seres vivos. Estas dificultades han sido estudiadas por diversos autores como Giordan (1984) o Banet y Núñez (1988-90).

Ambas apreciaciones se contradicen: una buena comprensión de la estructura celular de los seres vivos debería de facilitar la comprensión de los procesos fisiológicos, pero parece que no ocurre así. Ello nos hace sospechar que no se ha producido un aprendizaje significativo de este concepto (estructura celular de los seres vivos) puesto que el alumnado no es capaz de utilizario en situación de aplicación.

¿Cuál seráentonces el verdaderoobstáculoepistemológico? Posiblemente no se pueda encontrar una única respuesta. Nosotras creemos que una de los dificultades puede residir en que no se llega a entender bien cómo funciona la célula y por tanto cómo funciona un ser vivo en función de sus unidades celulares.

Además parece que la representación que tiene el alumnads: del funcionamiento interno de un ser vivo es similar al funcionamiento de una "máquina» o de un complejo «aparato» formado por piezas (corazón, estómago, etc.) y no se concibe como un proceso químico celular.

Otro aspecto que puede funcionar como obstáculo es ia concepción de que las células son piezas (ladrillos) del edificio físico de los seres vivos pero no unidades implicadas en los procesos biológicos.
Todos estos supuestos (hipótesis) son demasiado generales para una comprobación sistemática y rigurosa. Por ello, tomando esta idea general como punto de partida, nos ha parecido adecuado concretar una primera hipótesis operativa que podríamos resumir en el siguiente enunciado:

Los y las estudiantes al finalizar la enseñanza obligatoria tienen asumida la constitución estructural, con base celular de los seres vivos (en líneas generales), pero existe una gran dificultad a la hora de entender el funcionamiento de los organismos complejos como resultado del funcionamiento celular coordinado.

\section{DISEÑO EXPERIMENTAL}

\section{Estrategias de sondeo}

Los antecedentes bibliográficos consultados que abordan la probiemática de las ideas espontáneas de los alumnos y alumnas suelen proponer para explorar estas ideas diferentes tipos de cuestionarios. Las ventajas e inconvenientes de los diversos procedimientos (cuestionarios directivos, semidirectivos, entrevistas, observación en clase...) han sido señalados por varios autores (Giordan 1982).

Cuando el número de alumnos y alumnas es elevado, el cuestionario se muestra como un instrumento bastante interesante porque permite obtener una información amplia y sobre todo bastante homogénea, dada la cantidad de personas consultadas. Un sistema de entrevistas o de preguntas demasiado abiertas podía presentar ideas muy diversas y diff́cilmente categorizables.

Por tanto, decidimos plantear unas primeras cuestiones con preguntas directas que contestaron alumnos y alumnas de $8^{\circ}$ curso de EGB, esperando que el análisis de sus respuestas indicara líneas o características que permitieran perfilar nuestras hipótesis y elaborar un nuevo cuestionario que apuntara mejor en dirección a posibles obstáculos epistemológicos.

Del estudio y categorización de estas primeras respuestas pudimos obtener algunas ideas para la posterior realización de un sondeo de mayor relevancia, por ejemplo:

No tenía mucho sentido preguntar directamente si los seres vivos estaban formados por células porque la respuesta casi inequivocamente resultaba afirmativa y no aportaba ninguna luz al conocimiento de sus ideas previas ni de los obstáculos en el aprendizaje.

Muchas de las cuestiones formuladas se contestaban de forma que no ofrecían certeza ninguna a la hora de su análisis, citaremos únicamente dos ejemplos:

- La pregunta «;Todos los animales, vegetales y minerales están formados por células?» introducía con la palabra «mineral» una confusión en las respuestas ya que en varias ocasiones se detectó que el alumnado no 
había reparado en ella y contestaba en función del concepto de animal y vegetal.

- La pregunta « $i$ Todos los microorganismo están formados por células?» dio lugar a respuestas mayoritariamente afirmativas, pero sin mediar ninguna explicación, con lo que no podíamos averiguar si la respuesta se debía a la comprensión de que todo ser vivo está formado por células o a la idea de que éste era el caso de los microorganismos, pero no el de todos los seres vivos.

De estos primeros datos resaltaremos únicamente las diferencias entre las respuestas dadas acerca de la constitución celular de animales y de vegetales que comentaremos más adelante.

T'eniendo siempre presente que esta primera aproximación confirma un aspecto de nuestra hipótesis inicial en el sentido de que, al menos a nivel de formulación teórica, la mayoría del alumnado acepta que los seres vivos están formados por células, se elaboró un nuevo cucstionario donde se propusieron preguntas indirectas cuyas respuestas evidenciaran mucho más las ideas de los y las alumnas sobre el tema que nos ocupa y tuvieran una mayor incidencia en las implicaciones fisiológicas de la Teoría Celular.

Este cuestionario incluía:

a) Cuestiones directas que interpelan sobre la cstructura celular de los seres vivos (a efecto de mejor contrastar la primera parte de nuestra hipótesis).

b) Cuestiones indirectas sobre la estructura y funcionamiento de los seres vivos.

Estas cuestiones incluían la posibilidad de respuestas abiertas a fin de sondear mejor los esquemas mentales que las propiciaban.

\section{Características de la población}

Fueron consultados:

146 alumnos y alumnas de 8 EGB.

151 alumnos y alumnas de $1^{\underline{\sigma}}$ de BUP.

61 alumnos y alumnas de COU de Biología.

53 alumnos y alumnas de Escuelas de Formación de Profesorado.

Estos alumnos y aluınnas en ningún caso habían estudiado la célula o los tejidos durante cl curso en el que se encontraban, aunque si lo habían hecho (en todos los casos) en el curso anterior y también en todos los casos, habían cursado sus estudios con una metodología de trasmisión directa de conocimientos ya elaborados, como viene siendo usual en los centros docentes.

Por tanto la diferencia entre los grupos residía.

a) Fn el nivel de madurez intelectual.

b) Fin el grado de reiteración en cl estudio del tema: los alumnos/as de $8^{\circ}$ de EGB sólo habían trabajado este aspecto durante el curso anterior, los y las alumnas de $1^{\text {to }}$ de BUP lo habrían hecho en los dos cursos anteriores, y en caso de los y las alumnas de COU y Escuelas de Formación del Profesorado de EGB en tres cursos académicos.

Se han seleccionado distintos niveles educativos con el fin de contrastar mejor nuestra hipótesis general en el sentido de que, aun mediando reiteracion en el estudio del tema (un curso en el primer caso, dos para el segundo y tres para el tercero), los resultados no mostrarian diferencias importantes sobre todo en lo referente a la relación estructura/función, dado que no se habría producido (según nuestra hipótesis) un aprendizaje significativo en el período de instrucción escolar.

Para facilitar las respuestas, posibles interferencias entre ellas y evitar el cansancio a la hora de contestar el cuestionario, las preguntas se propusieron en dos fases separadas en el tiempo:

$1^{\underline{0}}$ Las preguntas relacionadas con la estructura celular.

$2^{\circ}$ Las preguntas relacionados con la función.

\section{RESULTADOS Y DISCUSIÓN}

En primer lugar se presentan los resultados de las cuestiones directas que se refieren a la estructura celular (apartado A). A continuación (apartado B) se presentan aquéllos que hacen referencia a respuestas sobre el funcionamiento, con base celular, de los seres vivos.

Se especifica el enunciado de cada pregunta, seguido de los resultados obtenidos en forma de tablas de porcentajes y desviación típica hallada, con una breve discusión de los mismos.

\section{A. Resultados que hacen referencia a la estructura celular de los seres vivos}

Pregunta: ¿Todos los animales están formados por células?

\section{Respuesta:}

Tabla I

Sobre si los animales están formados por células.

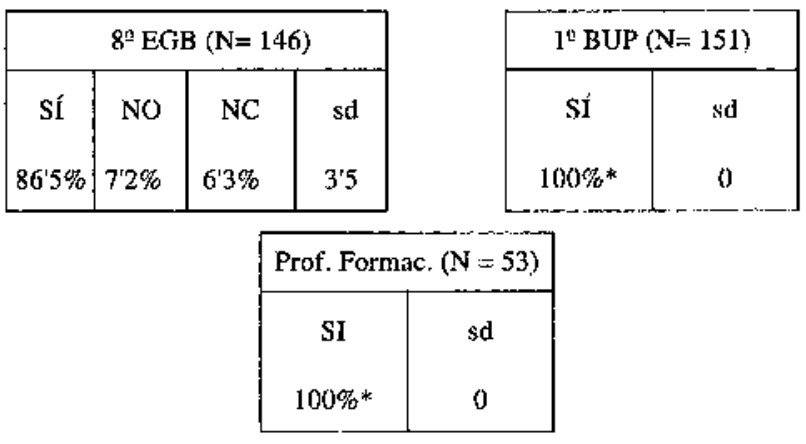

*Diferencias significativas al nivel de $0^{\prime} 05<\mathrm{D}>$. 
Pregunta: ¿Todos los vegetales están formados por células?

\section{Respuesta:}

Tabla II

Sobre si los vegetales están formados por células.

\begin{tabular}{|c|c|c|c|}
\hline \multicolumn{3}{|c|}{$8^{\circ} \mathrm{EGB} N=146$} \\
\hline SI & NO & NC & so \\
$59^{\prime} 4 \%$ & $174 \%$ & $20 \% \%$ & 5 \\
\hline
\end{tabular}

\begin{tabular}{|c|c|}
\hline \multicolumn{2}{|c|}{ Prof. Formac. $\mathrm{N}=53$} \\
\hline SI & sd \\
\hline $85 \% *$ & $49 \%$ \\
\hline
\end{tabular}

*Diferencias significativas al nivel de $0^{\prime} 05<\mathrm{D}>$

Pregunta: «De las cosas citadas a continuación đi cuáles tienen o son células...»

Respuesta:

Tábla III

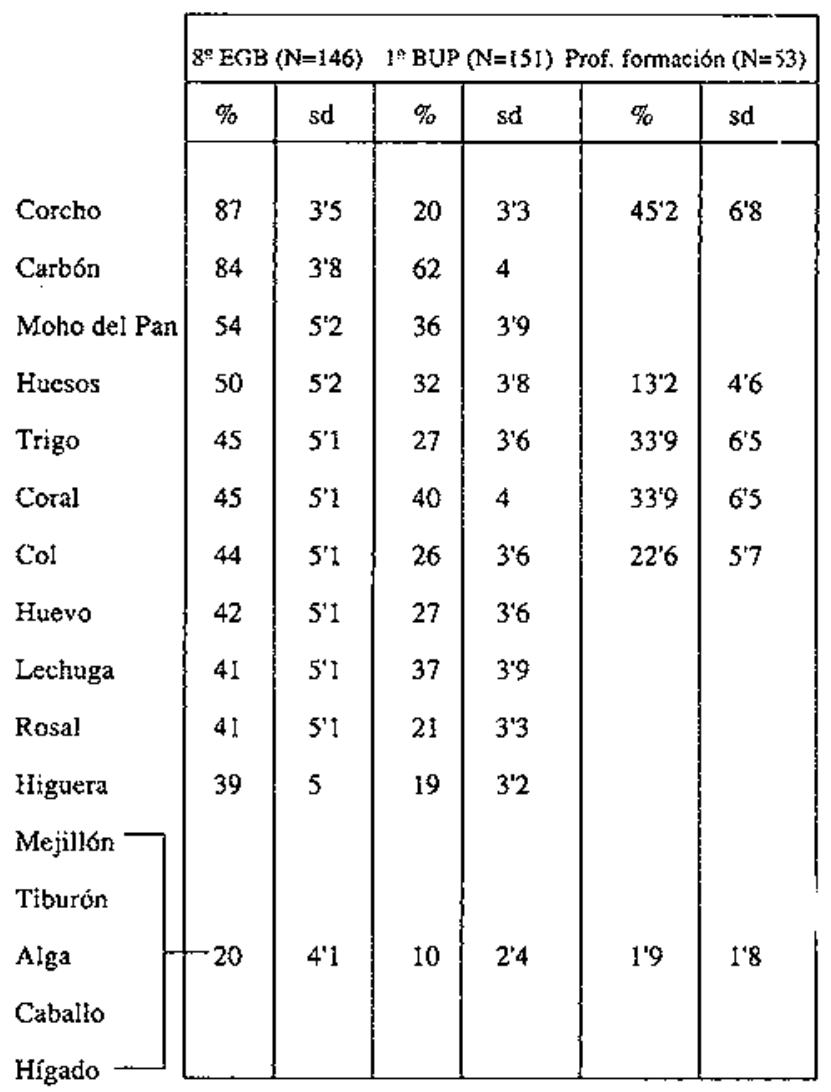

\section{Discusión}

Del análisis de los resultados obtenidos podemos extraer unas primeras ideas generales:

1) El conjunto de procesos de aprendizaje a lo largo de la vida escolar no consigue que los conocimientos se incorporen de forma estable y coherente; por ejemplo: cuando se pregunta si los animales están formados por células, las respuestas son afirmativas $\left(86^{\circ} 5 \%\right.$ en $8^{\circ}$ de EGB; $100 \%$ en los demás niveles), sin embargo esta afirmación es claramente incompatible con las respuestas dadas acerca de la estructura celular de los huesos, el trigo, la higuera, el mejillón...

Podríamos deducir a partir de estos porcentajes que la idea de que todos los seres vivos están formados por células se asume mentalmente, pero existen contradicciones que parecen mostrar que no se ha interiorizado significativamente el proceso; es decir:

Los alumnos y alumnas aparentemente creen o aceptan la idea de que los seres vivos están formados por células, pero, cuestionados en casos concretos o problemáticos, esta convicción se tambalea y se recurre a una respuesta aleatoria $i$ Qué mecanismo intelectual puede llevar a algunas personas a afirmar que un caballo o una higuera no están formados por células mientras que está segura de que sí lo están las manzanas o los tiburones? Esta respuesta carece de explicación lógica a no ser que pensemos en la duda, la vacilación y la respuesta dada al azar en los seres propuestos al final del listado.

2) Se observa una diferencia significativa entre las respuestas dadas respecto de las estructuras de los animales y de los vegetales.

Observamos que incluso cuando efectuamos las preguntas de forma directa son muchos más los alumnos y alumnas que han incorporado la idea de la estructura celular en los animales que en los vegetales.

$Y$ cuando nos atenemos a la estructura de seres vivos concretos (respondiendo a la tercera pregunta en la que se proporcionó una lista de nombres de cosas o seres para que señalaran aquéllos que tuvieran estructura celular), los errores más abundantes se cometen respecto de los vegetales.

Es bastante conocida la dificultad que tienen los niños y riñas pequeños en reconocer a los vegetales como seres vivos, dificultad que se arrastra un tanto en edades posteriores (Brumby 1982, Looft 1974, Tamir 1981, citados en las compilaciones de Jiménez y Serrano).

En este sentido, una asimilación automática y no significativa del concepto «ser vivo - formado por células» explicaría la dificultad en atribuir estructura celular a algo que no se tiene muy seguro como ser vivo.

3) Otros aspectos a destacar a partir de estos resultados es que aquellos seres vivos o partes de seres vivos que ofrecen un aspecto mineral, rígido... como huesos, corcho, coral... presentan mayor dificultad a la hora de 
entenderlos formados por células. Este aspecto pétreo o rígido no se asucia con la idea de vida, de ser vivo, y por tanto no se entiende como formado por células.

4) Todo ello nos lleva a pensar que nuestra hipótesis de partida de que los y las estudiantes adolescentes tienen asumida la constitución celular de los seres vivos desde el punto de vista estructural, se ve seriamente limitada cuando se debe aplicar a situaciones indirectas o de aplicación del concepto. Pensamos que ello se debe a que la asociación teórica «ser vivo - estructura celular» repetida tantas veces a lo largo de la vida académica no ha sido aprendida significativamente.

5) Fstadísticamente las diferencias entre los cursos de $8^{\circ}$ de $\mathrm{EGB}$ y los cursos superiores son significativas. Los alumnos y alumnas mayores cometen menos errores.

Atribuimos estos resultados a la maduración intelectual producida con la edad y la instrucción. Sin embargo los niveles de error, a nuestro parecer, todavía son altos. Éste sería un campo interesante de investigaciones futuras.

\section{B. Resultados que hacen referencia al funcionamien- to de los seres vivos (célula como unidad funcional)}

Pregunta: A veces nos arrancamos trozos de piel (cuando nos quemamos por el sol o cuando nos cortamos la cutícula de las uñas...). Si pudiéramos observar algunos de estos fragmentos al microscopio, ¿qué veríamos?

Respuestu: No relacionan la piel, y su regeneración, y su estructura con las células y, por tanto, no tienen clara la relación entre estructura y función

Tabla IV

Sobre la regeneración de la piel.

\begin{tabular}{|c|c|c|c|c|c|}
\hline \multicolumn{2}{|c|}{$80 \mathrm{VB}(\mathrm{N}=1 \overline{46})$} & \multicolumn{2}{|c|}{$1^{\mathrm{n}} \mathrm{BUP}(\mathrm{N}-151)$} & \multicolumn{2}{|c|}{$\operatorname{cov}(\mathrm{N}=61)$} \\
\hline$\%$ & $\mathrm{sod}$ & $\%$ & sd & $\%$ & $s d$ \\
\hline $30^{\prime} 8$ & 614 & 20 & 3,3 & 8: & $3 ' 5$ \\
\hline
\end{tabular}

\section{Discusión}

1) Los datos obtenidos muestran un aito porcentaje de personas que atribuyen estructura celular a la piel y la relacionan con su regeneración.

De las explicaciones que se dan en las respuestas deducimos que la representanción mental del alumnado está relacionada con la imagen «célula-ladrilıo» ligada al tejido epidérmico. Las respuestas nos hacen pensar que su esquema conceptual está más relacionado con un papel estructural de las células que con un papel funcional, lo que vendría a reforzar nuestra hipótesis inicial.

Las diferencias encontradas entre $8^{\circ}$ de $\mathrm{EGB}$ y $\mathrm{BUP}$ con respecto de COU resultan significativas estadísticamente.
Es decir, a medida que se progresa en edad e instrucción se afianza la idea de una base estructural de los orgttismos.

Pregunta: Los animales necesitan incorporar oxígeno a organismo. Esta función la realizan a través del aparato respiratorio y de allí el oxígeno pasa a la sangre y se distribuye por todo el cuerpo. ¿Por qué es necesaria esta distribución?

Respuesta: No relacionan el fenómeno de la distribución de oxígeno con la composición celular de los seres vivos (ni con las necesidades de cada célula...).

Tabia V

Sobre la distribución del oxígeno en el organismo.

\begin{tabular}{|c|c|c|c|c|c|}
\hline \multicolumn{2}{|c|}{$8^{\circ} \mathrm{EGB}(\mathrm{N}=146)$} & \multicolumn{2}{|c|}{$1^{\cap} \mathrm{BUP}(\mathrm{N}=151)$} & \multicolumn{2}{|c|}{$\mathrm{COU}(\mathrm{N}:=61)$} \\
\hline$\%$ & sd & $\%$ & sd & $\%$ & so \\
\hline 71 & $6^{\prime} 3$ & $61^{1} 6$ & 3,5 & 37 & 62 \\
\hline
\end{tabular}

\section{Discusión}

Cuando analizamos las respuestas dadas nos encontra mos con que resulta dificultoso relacionar esta distribución con la existencia de células, esto podría indicarnos que no se ha comprendido que las células, para desarro. Ilar sus procesos vitales están necesitadas de oxígeno y otras sustancias, lo cual estaría conectado con una idea inadecuada de lo que es un ser vivo.

Se entiende como ser vivo aquél que nace, crece, se reproduce y muere y no se liga el concepto con el de ciertas funciones metabólicas; por tanto, no se ve necesaria una distribución del oxígeno porque no es una necesidad para el ser vivo celular, sino para el ser vivo macroscópico.

La idea de que el oxígeno es necesario para el ser vivo, aunque sin saber ni por qué ni para qué, la sido bastatnte mencionada (el 65\% del alumnado de $1^{\circ}$ de BUP). También hemos encontrado una referencia frecuente a la necesidad de oxígeno de la sangre, sin mayor explicitación. Estos comentarios se podrían atribuir a la reiteración en el proceso de instrucción y los refuerzos constuntes de la vida cotidiana.

Atendiendo a los distintos niveles educativos en los que se ha realizado el sondeo podemos decir que las diferencias son significativas y a una mayor edau y madurez corresponden respuestas más adecuadas. Podríanos atribuir estas diferencias a que se conocen mejor los requisitos funcionales de la célula como ser vivo (necesidad de oxígeno y nutrientes...) y, por tanto, se es consciente de la necesidad de tuna organización en los organismos complejos en relación con estas necesidiades celulares (no olvidemos que los y Ias alumnas de COU sí han estudiado un metabolismo celular básico en el curso académico anterior). A pesar de ello, casi cl $40 \%$ del alumnado de $\mathrm{COL}$ no relaciona el fenómeno de la distri- 
bución de oxígeno con la composición celutar de los seres vivos.

Pregunta: Algunas plantas como los geranios se plantan en los jardines mediante trozos de tallos que se ponen en el suelo. ¿Cómo es posible que, a partir de esos trozos vuelva a desarroliarse un geranio entero?

Respuesta: No relacionan el crecimiento de plantas con la estructura ni la proliferación celular.

Tabla VI

Sobre el creciniento de las plantas.

\begin{tabular}{|c|c|c|c|c|c|}
\hline \multicolumn{2}{|c|}{$8 \mathrm{EGB}(\mathrm{N}=146)$} & \multicolumn{2}{|c|}{$1^{\circ} \overline{B U P}(\mathrm{~N}=151)$} & \multicolumn{2}{|c|}{$\operatorname{COU}(N=61)$} \\
\hline$\%$ & sd & $\%$ & sd & $\%$ & $s d$ \\
\hline $75^{\prime} 6$ & 67 & $86^{\prime} 8$ & 28 & $64 *$ & 61 \\
\hline
\end{tabular}

\section{Discusión}

Podemos interpretar que, aunque se ha contestado mayoritariamente en otros cuestionarios que las célu'.as se reproducen, la idea de la reproducción podría ir ligada a la conservación de la especie celular (microorganismos) o a la reproducción de un ser complejo (para dar otro individuo complejo), pero no estaría vinculada con el crecimiento de un organismo, al menos con el crecimiento de los vegetales.

Por tanto, el aprendizaje realizado, sobre lo que es la base celular del crecimiento en un ser complejo, no es significativo.

De los comentarios vertidos en las respuestas podemos inducir que un $90 \%$ de los y las estudiantes consultados conocen que los vegetales tienen un proceso especifico de regeneración, que tienen un tipo de reproduczión vegetativa, que pueden aparecer plantas nuevas a partir de fragmentos de tallos, etc., pero nunca lo asocian a ningún proceso de reproducción celular.

También hay que señalar la diferencia significativa encontrada entre las respuestas dadas por los y las alumnas de COU y los restantes niveles. Aunque la cantidad de respuestas inadecuadas $(64 \%)$ en COU es muy elevada, se puede asegurar que tos resultados son mucho mejores que en $1^{\circ}$ de BUP y $8^{\circ}$ de EGB. Ello sería coherente zon los argumentos de Dreyffus y Jungwith (ya citados) en el sentido de que un mejor conocimiento del funcionamiento bioquímico celular contribuye a la comprensión de la célula como ser vivo y como integrante de los organismos más complejos $y$, por tanto, de las relaciones entre estructura y función que se derivan.

Pregunta: Algunos órganos, como por ejemplo las glándulas salivares, producen unas sustancias que facilitan el funcionamiento de nuestro cuerpo, en este caso, la digestión de los alimentos. Explica quién fabrica saliva en las glándulas salivares.

Respuesta: No atribuyen el funcionamiento de las giándulas a la acción individual y coordinada de las células constituyentes.

Tabla VII

Sobre la secreción de las glándulas.

\begin{tabular}{|c|c|c|c|c|c|}
\hline \multicolumn{2}{|c|}{$8^{\circ} \mathrm{EGB}(\mathrm{N}=146)$} \\
\hline$\%$ & $\mathrm{sd}$ \\
\hline 927 & $4^{\prime} 1$ & $\%$ & $\mathrm{Cd}$ & $\%$ & $\mathrm{COU}(\mathrm{N}-61)$ \\
\hline 925 & 22 & 859 & 48 \\
\hline
\end{tabular}

\section{Discusión}

En este caso, el porcentaje de respuestas inadecuadas $\mathrm{cs}$ significativamente mayor que en las preguntas anteriores. No hay diferencias significativas entre grupos. En los tres casos ha habido muy pocos alumnos y alumnas que hayan realizado la correspondencia celular-función de una glándula del organismo complejo.

Estos resultados de hecho reiteran nuestra hipótesis, pues la complejidad del proceso sobre el que se interroga es muy grande.

Hemos visto que las respuestas referentes a la necesidad de la distribución de oxígeno eran ya bastantes inadecuadas, porque no sc entendía esta necesidad celular $y$, por tanto, no se podía comprender que los organismos complejos necesitaran estos mecanismos de distribución; mucho más difícil será el comprender que las células, además de automantenerse, elaboran productos o ejercen funciones que no están ligados a su propio mantenimiento sino a las necesidades de un ser vivo (necesidades que no se ven relacionadas con Ia propia célula, sino con un ente completo y macroscópico), sobre todo si tenemos en cuenta que desde que se comienza en la escuela, se trabajan Ias funciones del organismo (respiración, movimiento, reproducción...) como algo que realizan determinados aparatos, «partes» de ese organismo (como el funcionamiento de una máquina). Únicamente a los 12 años se comienza a introducir el concepto de célula y pluricelularidad.

A pesar de todo, en el proceso posterior a los 12 años, los resultados obtenidos en COL y en los profesores en formación parecen indicar que tampoco se produce aprendizaje significativo de estos conceptos cuando media una metodología de transmisión verbal.

Pregunta: Cuando los niños y las niñas son pequeños, sus huesos son de pequeño tamaño. A medida que se hacen mayores, los huesos van creciendo. Explica cómo es el mecanismo del crecimiento de los huesos.

Respuesta: No atribuyen el crecimiento de los huesos a una proliferación celular. 
Tabla VIII

Sobre el crecimiento de tos huesos.

\begin{tabular}{|c|c|c|c|c|c|}
\hline \multicolumn{2}{|c|}{$8^{\circ} \mathrm{EGB}(\mathrm{N}=146)$} & \multicolumn{3}{|c|}{$1^{\circ} \mathrm{BUP}(\mathrm{N}=151)$} \\
\hline$\%$ & sd & \multicolumn{2}{|c|}{$\mathrm{COU}(\mathrm{N}=61)$} \\
\hline $92^{\prime} 7$ & 41 \\
\hline
\end{tabular}

\section{Discusión}

La idea del crecimiento de los huesos no está ligada a una estructura celular; prácticamente se puede decir que en ningún caso y en ningún nivel, puesto que las diferencias entre los grupos no han resultado significativas.

Además, esta pregunta registra cl más alto nivel de respuestas inadecuadas de cuantas se han formulado.

Atribuimos estos resultados a dos factores:

a) La dificultad ya mencionada de entender el crecimiento como resultado de la proliferación celular.

b) El aspecto pétreo o mineral de los huesos que no permite la conexión con la idea de ser vivo. Si no se considera que el hueso está formado por materia viva, es lógíco que se piense que no está formado por células. Estos resultados los podemos conectar con los obtenidos en el primer apartado del cuestionario.

\section{CONCLUSIONES}

Despućs de las discusiones anteriores podemos extraer dos tipos de conclusiones:

\section{A. Respecto de la estructura celular de los seres vivos}

Aunque la mayor parte de las respuestas confirman nuestra hipótesis de partida de que el alumnado ha asumido de forma teórica que los seres vivos están formados por células y contestan adecuadamente cuando se les pregunta directamente sobre ello, Ilama la atención que un porcentaje no despreciable de la población de $8^{\mathrm{g}}$ de $\mathrm{EGB}$ no responda rotundamente y vacile en el caso de los vegetales.

Las contradicciones encontradas parecen mostrar que este concepto no se ha interiorizado significativamente atun en el caso de personas de mayor edad (entre un 20 y un $30 \%$ de los y las alumnos y alumnas de Escuclas de Formación del Profesorado).

En este aspecto veríamos falseada parte de nuestra hipótesis inicial.

\section{B. Respecto de la base celular en el funcionamiento fisiológico de los organismos}

Aun cuando se asuma la estructura celular, no se relaciona con funciones físiológicas tales como el transporte, el crecimiento o las funciones de secreción, to cual apoya nuestra hipótesis.

Parece ser que existe una gradación de dificultad a la hora de cntender determinadas funciones de los organis. mos complejos: son más fáciles de entender los procesos derivados de las necesidades de las células constituyentes (como el transporte de oxígeno) que los procesos derivados del servicio de las células al funcionamiento global del organismo (crecimiento o secreción).

De todo ello podemos concluir que nucstra hipótesis de que «existe una gran dificultad a la hora de entender el funcionamiento de los organismos complejos como resultado del funcionamiento celular coordinado» se ve confirmada en este primer trabajo mientras que la sasunción de que existe una constitución celular en todos los seres vivos» se ha visto falseada para el caso de los vegetales.

\section{PERSPECTIVAS}

1) Comprender que los seres vivos están formados por células no es algo que resulte fácil puesto que no es un fenómeno observable directamente ni de manera cotidiana.

Históricamente el proceso de elaboración de la teoría celular ha durado muchísimo tiempo y sólo ha sido posible a partir de importantes avances tecnológicos que han permitido la observación directa de las células que forman parte del organismo (además del importante cambio que supuso la incorporación de la metodología científica hipotético-deductiva).

Durante el período de instrucción general básica el alumnado apenas desarrolla actividades prácticas en las que se observe, se reflexione y discuta acerca de la estructura celular de los seres, sólo se les insiste en la idea de forma reiterada. Recordemos que a estas edades es muy importante apoyar el aprendizaje en la percepción y proporcionar imágenes visuales que permitan elaborar modelos gráficos o fotográficos, pero en la mayoría de las escuelas no se realizan actividades de este tipo (muchas de ellas no tienen o no utilizan microscopios, fotografías o diapositivas). ¿Podría esta deficiencia es" tar relacionada con la dificultad de aprehensión de este conocimiento? Éste sería uno de los campos a investigar como derivación de estos primeros resultados.

2) Parece ser que una de las mayores dificultades a la hora de cntender a los seres vivos como constituidos estructural y funcionalmente por unidades celulares es la de comprender adecuadamente las características que definen al ser vivo. Se tiende a pensar que es algo que 
funciona macroscópicamente, como una serie đe máquinas acopladas, y no se atribuyen necesidades de materia y energía a cada una de las células, como si éstas no necesitaran cubrir necesidades vitales.

El nivel de comprensión y aceptación de la célula como unidad funcional es muy bajo en todos los niveles estudiados. De acuerdo con Dreyffus y Jungwith (ya citados) esto constituye un verdadero obstáculo epistemológico para comprender el funcionamiento del organismo complejo. Insisten en la idea de que una de las dificultades para entender la célula como ser vivo reside en el desconocimiento de los procesos a nivel bioquímico de los procesos biológicos y por ello recomiendan expresamente que estos contenidos se traten postcriormente.

Otra derivación de esta primera aproximación a las ideas sobre la constitución celular de los seres vivos será, pues, investigar sobre estas dificultades y sobre si es verdaderamente difícil la vía del conocimiento de niveles químicos complejos o puede superarse con una metodología distinta este obstáculo epistemológico.

3) De acuerdo con la literatura de los últimos años, una propuesta de enseñanza aprendizaje de las Ciencias naturales que favorezca la superación de las ideas intujtivas de los y las alumnos y alumnas, y que afiance los conceptos, pasa necesariamente por procedimientos y actitudes de cambio, de análisis crítico... promovidos a través de actividades coherentes con la naturaleza del trabajo científico.

A nuestro entender los rasgos generales de una proptesta metodológica para el estuđio de este tema deberían

\section{REFERENCIAS BIBLIOGRÁFICAS}

BANET, E. y NÚNẼZZ, F, 1988. Ideas de los alumnos sobre la digestión: Aspectos anatómicos, Énseñanza de las Ciencias, Vol. 6(1), pp. 30-37.

BANET, E. y NÚÑEZ, F., 1990. Esquemas conceptuales de los alumnos sobre la respiración, Enseñanza de las Ciencias, Vol. 8(2), pp. 105-111.

BELL, B., 1981. What is a plant? Some children ideas, n. 2, Science Teacher, 31, pp. 10-14.

BRUMBY, M., 1979. Problems in learning the concept of natural selection, J. Biological Education, 13, pp. 119-122.

BRUMBY, M., 1982. Students perceptions of the concept of life, Science Fducation, 66 (4), pp. 613-622.

CAÑAI., P., 1986: La nutrición vegetal, un año después. Un estudio de caso en séptimo de $\mathrm{EGB}$, Investigación en la Escuela, 3, p. 55 .

CAÑAL, P., 1986. Las representaciones de los alumnos: ‘exrores a eliminar o pasos necesarios en el proceso evolutivo de reconstrucción personal del conocimiento? Actas de las. $I V$ contemplar aspectos mencionados en los dos apartados anteriores, como por ejemplo:

1) Promover la explicitación de las ideas espontáneas de las ahumnas y los alumnos.

2) Con las ideas que proporcionen, se diseñarán experiencias que permitan comprobar o refutar sus ideas.

3) Proveer de imágenes reales de la estructura celular de los seres vivos que faciliten una elaboración mental de los modelos biológicos.

4) Planteamiento de problemas acerca de la estructura celular de los seres vivos.

5) Elaboración de una secuencia de actividades en las que se planteen los conflictos cognitivos pertinentes, utilizando como apoyo las investigaciones realizadas, las ideas expresadas por los alumnos y las alumnas y la historia del proceso de elaboración de la teoría celular.

Nos parece necesario, para terminar, comentar que cualquier secuencia de trabajo no debería pretender terminar con el problema de los «errores conceptuales» que pudieran tener los o las alumnas, más bien se trataría de poner las bases para que este concepto no se comportara como un obstáculo epistemológico y pudiera abordarse de nuevo la relación estructura/función a nivel celular en etapas posteriores facilitando, por tanto, el aprendizaje en cursos posteriores cuando existan ya unas nociones de química y puedan reinterpretarse estos conocimientos básicos.
Jornadas de Investigación en la Escuela, Sevilla, pp. 133138.

CARRASCOSA, צ., 1985. Errores conceptuales en la Enseñanza de Ia Física y Química: una revisión bibliográfica, $E n$ señanza de las Ciencias, Vol. 3(3), pp. 230-234.

DREYFUS y JUNGWITH, 1988. The cell concept of 10 th graders: curricular expectations and reality, International Journal of Science Education, Vol. 10, pp. 221-230.

GAGLIARDI, R. et al., 1987. Nous ne sommes pas des petits ballons. Les modeles spontanés des éleves de l'école italiennc sur la célfule. Actes IXèmes Journées Internationales sur $l^{*}$ education Scientifique. Chamonix.

GENÉ, A. y ASTUDILLO, H., 1984. Errores conceptuales en Biologia: la fotosíntesis de las plantas verdes, Enseñanza de las Ciencias, Vol. 2 (1), pp. 15-16.

GIORDAN, A., 1978. Observations-experimentation: mais comment les ếlèves apprennent-ils?, Revue Francaise de Pedagogie, 44, pp. 66-73. 
GIORDAN, A., 1982, La Enseñanza de las Ciencias. (Siglo XXI: Madrid).

GIORDAN, A., 1984. Comprehesion de livres scientifiques de vulgarisation (text et iconographie) par des enfants de 8 à 14 ans. Actes VI Journées Internationales sur L'éducation scientifique, pp. 661-670.

GIORDAN, A., 1989. De las concepciones de los alumnos a un modelo de apredizaje alostérico, Investigación en la Escuela, 8, pp. 3-13.

GIORDAN , A. y MARTINAND, J.L., 1988. Étât des recherches sur les conceptions del éléves en Biologic. (Autoedición: París).
JIMÉNEZ, M.P., 1987. Preconceptos y esquemas conceptuales en Biología. Selección bibliográfica, Enseñanza de las Ciencias, 5 (2), pp. 165-167.

LOOFT, W.R., 1974. Animistic thougth in children: understandig of eliving» across its associated attributes, Journal of Genetic Psych, 124, pp. 235-240.

SERRANO, T., 1987. Representaciones de los alumnos en Biologia: estado de la cuestión y problemas para su investigación en el aula. Actas de las IV Jornadas de Investigación en la Escuela. Sevilfa, pp. 157-161. 